\title{
Rheumatology university faculty opinion on coronavirus disease-19 (COVID-19) vaccines: the vaXurvey study from Egypt
}

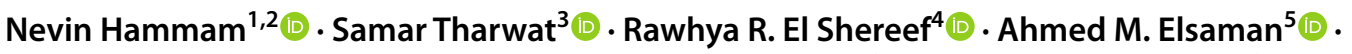

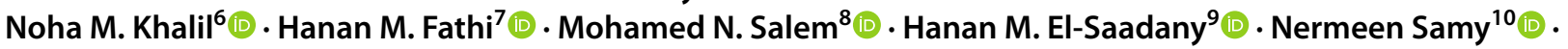

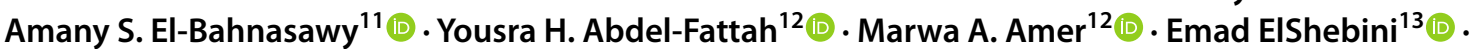

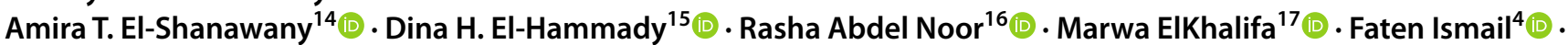

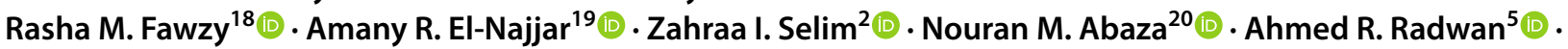

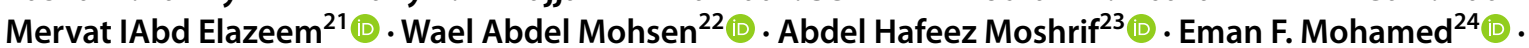

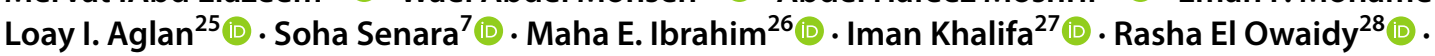 \\ Noha El. Fakharany ${ }^{29}$ (]) Reem Hamdy A. Mohammed ${ }^{29}$ (I) $\cdot$ Tamer A. Gheita ${ }^{29}$ (i) on behalf of the Egyptian College \\ of Rheumatology (ECR) COVID-19 Study Group
}

Received: 31 May 2021 / Accepted: 28 June 2021 / Published online: 9 July 2021

(c) The Author(s), under exclusive licence to Springer-Verlag GmbH Germany, part of Springer Nature 2021

\begin{abstract}
Objectives The aim of the present work was to explore the perspectives of Egyptian Rheumatology staff members as regards the coronavirus disease-19 (COVID-19) vaccine.

Methods The survey is composed of 25 questions. Some questions were adapted from the global rheumatology alliance COVID-19 survey for patients.

Results 187 rheumatology staff members across Egypt from 18 universities and authorizations actively participated with a valid response. The mean time needed to complete the survey was $17.7 \pm 13$ min. Participants were 159 (85\%) females (F:M 5.7:1). One-third agreed that they will be vaccinated once available, $24.6 \%$ have already received at least one dose, $29.4 \%$ are unsure while $16 \%$ will not take it. Furthermore, $70.1 \%$ agreed that they will recommend it to the rheumatic diseases (RD) patients once available, $24.1 \%$ are not sure while $5.9 \%$ will not recommend it. RD priority to be vaccinated against COVID-19 in descending order include SLE (82.9\%), RA (55.1\%), vasculitis (51.3\%), systemic sclerosis (39.6\%), MCTD (31.6\%), Behcet's disease (28.3\%). The most common drugs to be avoided before vaccination included biologics $(71.7 \%)$, DMARDs (44.4\%), biosimilars (26.7\%), IVIg (17.1\%) and NSAIDs $(9.1 \%)$.

Conclusions The results of the study and specifically the low rate of acceptability are alarming to Egyptian health authorities and should stir further interventions to reduce the levels of vaccine hesitancy. As rheumatic disease patients in Egypt were not systematically provided with the vaccine till present, making the vaccine available could as well enhance vaccine acceptance. Further studies to investigate any possible side effects, on a large scale of RD patients are warranted.
\end{abstract}

Keywords Coronavirus disease-19 (COVID-19) · Vaccines · Egyptian $\cdot$ Rheumatology staff members

\section{Introduction}

The Coronavirus disease 2019 (COVID-19) pandemic is an international worry caused by severe acute respiratory syndrome coronavirus 2 (SARS-CoV-2) [1, 2]. It has hit nearly every corner of the world and changed the face of medicine

Tamer A. Gheita

gheitamer@hotmail.com

Extended author information available on the last page of the article
[3]. At the time of the study, 174,209,885 confirmed cases are affected worldwide with a mortality of 3,747,389 cases. The official reports for confirmed cases in Egypt are 267,972 cases and 15,352 deaths $[4,5]$. The most common symptoms were fever and cough while diarrhea was uncommon. Ground-glass opacity was the prominent radiologic finding on chest computed tomography (CT) [6]. Despite that, most COVID-19 patients were afebrile, and many did not have abnormal radiologic findings. The fundamental cardio-respiratory illness and co-morbidities such as hypertension and 
diabetes mellitus tend to amplify the severity of COVID19 [7]. Rheumatic manifestations such as arthralgias and arthritis are noticeable in $15 \%$ of cases [1]. With the disease progress, a cytokine storm may evolve with consequent fatal complications. In addition to the basic therapeutic options including ventilatory support, antiviral drugs, symptomatic and complementary remedies, some anti-rheumatic drugs have a likely role [2].

During the pandemic, rheumatologists agreed to their key emerging frontline role in treating COVID-19. Rheumatologists by far manage outcomes of immune system dysfunction and are user friendly with using immune modulators which paves way for their key contribution in the multidisciplinary management of COVID-19 patients. Likewise, rheumatologists have to wait and see whether SARS-CoV-2 infection triggers autoimmunity in patients with COVID-19 infection in the future [8]. In a recent study conducted by the Egyptian College of Rheumatology (ECR) COVID-19 study group, potential changes in rheumatology practice by staff members evolved since the COVID-19 pandemic [9]. The challenges of the pandemic have hastened changes in the way rheumatologist deliver health care [10]. Rheumatic diseases (RDs) constitute a susceptible set [11]. It has been suggested that RD patients should sustain immunosuppressives, avoid infection by social distancing and halt immunosuppressants in case of infection [2]. The Global rheumatology Alliance (GRA) registry reported that the incidence of infection is greater among RD patients with a possible relation to prednisone dose of $>10 \mathrm{mg} /$ day with no difference between patients treated with biologic or targeted disease-modifying anti-rheumatic drugs (DMARDs) $[12,13]$.

The scientific and larger pharmaceutical communities have been tasked with the development, testing, and production of a safe and effective vaccine as a longer-term solution to prevent further spread and recurrence of COVID-19 infection throughout the populace [14]. There is an abundance of evidence indicating how the different vaccines stimulate the immune response [15] and it is presumed that an effective vaccination against COVID-19 could mobilize the innate and adaptive immune responses [16]. After the binding of the viral surface spike protein with a human cell receptor, the viral membrane fuses with that of the human cell, thus allowing viral genome to enter the cell. Once inside, the viral RNA is translated, and more viruses are replicated. Antigen-presenting cells (APCs) ingest the virus and display the viral peptide, consequently activating $\mathrm{T}$ helper cells, which stimulate B cells to make antibodies against the virus. A similar immune response occurs with the administration of the different types of vaccine [15].

Vaccines against COVID-19 optimally protect by provoking amplified quantities of high affinity virus-neutralizing antibodies and may shield against severe forms and prevent adverse effects $[16,17]$. In spite of the development of vaccines to neutralize the virus and with the lack of explicit treatments, the use of immunomodulatory drugs could be sensible in severe cases and early management of complications allows for a more complete recovery [18]. Yet, studies on the vaccine necessitate precise clinical management, aided with thorough evaluation of safety and immune responses [17]. It has been pointed to the importance of the vaccine as reduced uptake may lead to outbreaks with consequent necessity of local lockdowns and a hampered pandemic response [19]. Nevertheless, in light of the third wave much uncertainty remains. In this regard, the COVID-19 vaccine is readily expected to be one of the leading solutions to the pandemic. Emergence of several potential vaccines has been the topic of discussion, also in Egypt. Most proposed COVID-19 vaccines are two dose regimens, and the second dose will hopefully cover those patients who did not produce a strong immune response with the first dose [20]. Whilst the safety and efficacy of the vaccines are mostly reassuring, reports of unfavorable immunological reactions to the vaccine may be of primary concern to patients with autoimmune diseases with limited information [3]. The aim of the present work was to study the perspectives of Egyptian rheumatology staff members across the country as regards the COVID-19 vaccines.

\section{Methods}

The survey included 25 questions distributed by emails through a specially designed google form (using the Microsoft Network; msn web portal) over 1 week during April 2021, to University staff members across Egypt representing 26 rheumatology or internal medicine and pediatrics (rheumatology division) departments from 18 Universities and authorizations that include 600 rheumatology staff members. Participating staff were also members of the ECR study group.

The questions were mainly in the format of checkboxes, dropdown or short answers. The survey was designed so as to be easily filled in by the participating staff member. In an attempt to validate the survey, a pilot test was run and a revision of the survey design, content, terminologies, comprehension and easiness to fill-in was performed by a group of rheumatology staff members. The questions were mostly close ended (only the options for 'others' were open-ended) and the response was according to the mentioned instructions. Answers were recorded by receiving the filled in survey (google forms) and were transferring into an excel sheet. A written consent was provided by the participating Rheumatology University staff members. The study questionnaire/survey conforms to the local ethical committees of the corresponding universities and is in accordance with the Declaration of Helsinki.This work was conducted 
in accordance to the recently published primer for author guidelines for reporting survey-based studies [21].

Some questions were adapted from the Global Rheumatology Alliance (GRA) COVID-19 Vax Survey for patients [22] that is officially supported by the ECR. The questions provided on the original GRA survey are 78 questions. The survey is in English language and presented in Appendix 1.

The areas covered by the survey include questions that assess the rheumatology staff members' perspectives on the current status of the available vaccines for the rheumatology staff members as well as their viewpoint for the RD patients. After the participant fills in his/her basic information there were 25 questions. Question 1 focused on the perspective of the rheumatologists on the most vulnerable RDs that should be vaccinated with superiority. Then question 2 considered the medications that would rather be temporarily stopped or avoided for a RD pre- vaccination. The co-morbidities that would halt the treating rheumatologist from considering vaccination of his/her patient constituted question 3. Questions 4-9 cover the willingness of the rheumatologist to get vaccinated or have his /her RD patients receiving the vaccine too. Question 10 is concerned about the sources of information that would influence the decision that the rheumatologist gets vaccinated. Question 11 includes 14 statements related to the concerns about the COVID-19 vaccine for the rheumatologist and/or the RD patients. Furthermore, question 12 includes 8 statements regarding how to overcome the pandemic. Question 13 points to the likelihood of getting infected by COVID-19 and question 14 (3 statements) is on the approach of the rheumatologist in case the RD patients get vaccinated. Questions 15-17 are on the possibility that RD patients got vaccinated by other vaccines (else that the COVID-19) and if there was any report for serious side effects. Question 18 includes 14 side effects that may have occurred with rheumatology staff members as well as whether or not there was affection of the RD activity or development of new ones reported. Question 19 targets the likeliness that the rheumatologist would change the treatment plan for a RD patient who got vaccinated if he/she has a flare or worsening of the condition. Questions 20-24 are about the frequency of rheumatology staff members who got infected or vaccinated and which vaccine would be preferable also for the RD patients. The last question points to the greatest concern about temporarily stopping the medications for $\mathrm{RD}$ patients to give them the COVID-19 vaccine.

\section{Statistical analysis}

Statistical package for social science (SPSS version 24) was used for data analysis. The results were presented as number and percentage. Chi-Square and ANOVA tests were performed to compare 2 or more groups respectively. $p<0.05$ was considered significant.

\section{Results}

\section{Characteristics of the respondents}

In this study, out of 195 submitted responses, 187 rheumatology staff members actively participated by filling out the form with a valid response. Among 153 participants who recorded the time, the mean time needed to complete the survey was $17.7 \pm 13 \mathrm{~min}$. The frequency of participation was $31.17 \%$ (187/600). They were 159 (85) females and 28 (15\%) males (F:M 5.7:1). The contribution was from the following Universities: Minia $13.4 \%$, Cairo (12.8\%), Mansoura (9.1\%), Assuit (8.6\%), Aswan (7.5\%), Zagazig and Suez Canal (5.3\% each), Alexandria, Helwan and Ain Shams (4.8\% each), Benha (4.3\%), Al-Azhar (3.7\%), Sohag, Tanta and Fayoum (3.2\% each), Beni-Suef (2.7\%), Menoufiya (2.1\%) and South-valley (1.1\%). Staff members' specialties were 25 internal medicine (rheumatology divisions), 159 rheumatology and rehabilitation and 3 pediatric rheumatologists. The experience as a rheumatologist was $11.7 \pm 8.7$ years (ranging from 0.7 to 42 years). The majority were assistant lecturers (47.1\%), $20.9 \%$ were lecturers and $15.5 \%$ assistant professors while $16.6 \%$ were professors.

\section{Willingness of the rheumatologist to the vaccine}

One-third (30.5\%) of the respondents agreed that they will be vaccinated once available, $24.6 \%$ have already received at least one dose, $29.4 \%$ are unsure while $16 \%$ will not take it. Furthermore, $70.1 \%$ agreed that they will recommend the vaccine to the RD patients once available, $24.1 \%$ are not sure while $5.9 \%$ will not vaccinate them. The response to the survey as regards the priority of RD to be vaccinated, the medications rather to be avoided for patients before vaccination and the co-morbidities that may defer the decision to vaccinate are presented in Figs. 1 and 2.

As for the factors that may increase the willingness to be vaccinated, most of the rheumatology staff members (69\%) need more information, $27.8 \%$ will be vaccinated if someone trusty gets it first, $24.6 \%$ need to see more people have had it to judge, $13.9 \%$ if more vulnerable people get vaccinated first and 5.3\% reported that nothing will increase their willingness. $2.1 \%$ reported other factors such as the need to have a vaccine certificate for traveling abroad, the necessity to perform investigations that assess thrombotic potential before being vaccinated and the duration of protection provided by the vaccine was a concern. 


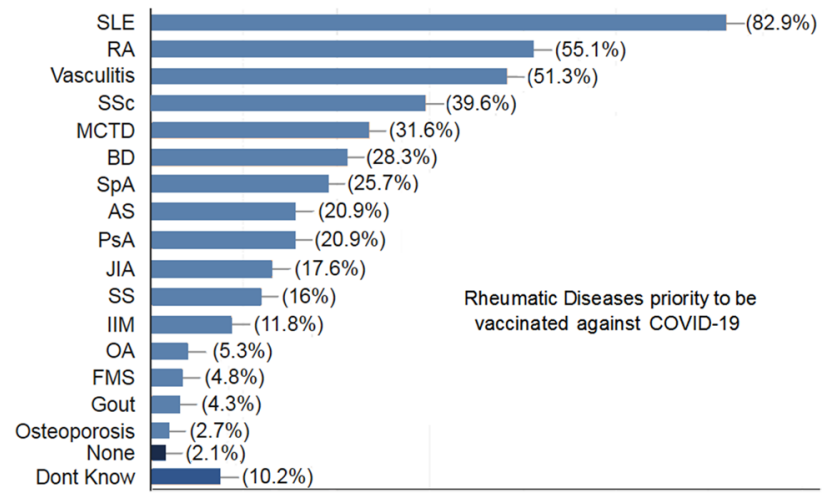

Fig. 1 The Rheumatology staff members' opinion on the rheumatic diseases (RD) with priority to be vaccinated. $S L E$ systemic lupus erythematosus, $R A$ rheumatoid arthritis, $S S c$ systemic sclerosis, MCTD mixed connective tissue disease, $B D$ Behcets disease, $S p A$ spondyloarthritis, $A S$ ankylosing spondylitis, $P S A$ psoriatic arthritis, JIA juvenile idiopathic arthritis, SS Sjogrens syndrome, IIM idiopathic inflammatory myopathy, $O A$ osteoarthritis, FMS fibromyalgia syndrome

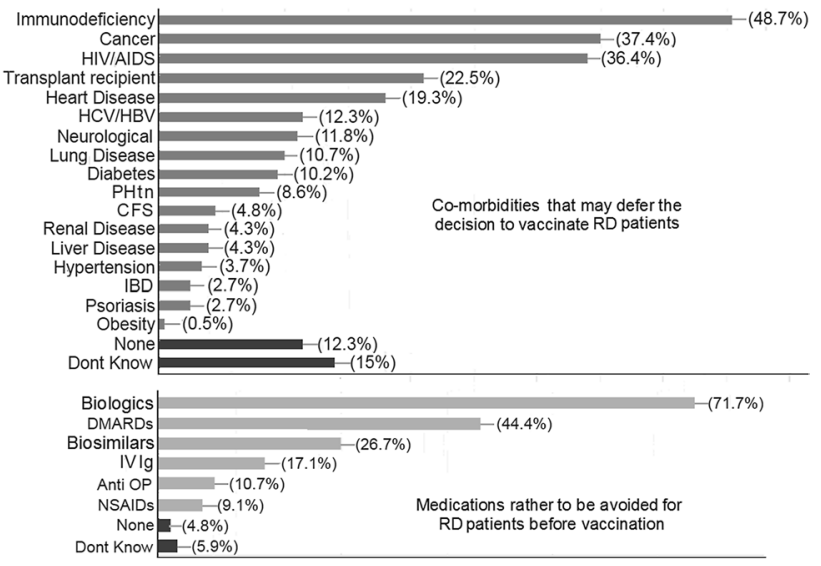

Fig. 2 The Rheumatology staff members' opinion on the co-morbidities that may defer the decision to vaccinate rheumatic diseases (RD) patients and the medications to be rather avoided for RD patients before vaccination. HIV/AIDS human immunodeficiency virus/ acquired immunodeficiency syndrome, $H C V / H B V$ hepatitis $\mathrm{C}$ and $\mathrm{B}$ virus, $P H t n$ pulmonary hypertension, $C F S$ chronic fatigue syndrome, $I B D$ inflammatory bowel disease, DMARDs disease-modifying antirheumatic disease, IV Ig intravenous immunoglobulin, $O P$ osteoporosis, NSAIDs non-steroidal anti-inflammatory drugs

Regarding the RD patients, more information is required by $76.5 \%$ to consider the vaccine by the rheumatology staff members, $34.8 \%$ will be in favor of vaccination if other RD patients get it first, $28.3 \%$ if other highly vulnerable patients are vaccinated, $19 \%$ will consider it if the patient asks for it, $17.9 \%$ if the health condition and activity of their RD patients improves, $4.9 \%$ will consider it with any change in the medications of their patients, while nothing will increase the willingness in $2.7 \% .1 .1 \%$ reported other factors as the need for knowing the thrombotic potential of the patient before being vaccinated.

\section{Source of information about the vaccine}

Regarding the sources that are most likely to influence the decision to receive the vaccine, the majority $(76.8 \%)$ will rely on professional organizations. Less likely to rely on news media and social media (7.2 and 3.3\%; respectively).

Regarding the response to different statements related to the concerns about the COVID-19 vaccine for the rheumatologist and/or the RD patients and how to overcome the pandemic; questions 11 and 12, see Figs. 3 and 4. 24.2\% are neutral and $24.8 \%$ don't know if COVID-19 vaccine will lead to serious side effects, $13.3 \%$ agree, $6.7 \%$ strongly agree, $16.4 \%$ disagree and $14.5 \%$ strongly disagree.

$29.4 \%$ agree to stop regular RD medications for a short time to take the vaccine, $7.5 \%$ strongly agree, $20.9 \%$ are neutral, $18.2 \%$ disagree, $13.4 \%$ strongly disagree and $10.7 \%$ don't know. $33.2 \%$ are neutral to whether the vaccine will lead to a disease flare, $21.9 \%$ disagree, $12.8 \%$ strongly disagree, $8.6 \%$ agree and $3.2 \%$ strongly agree while $20.3 \%$ don't know. $20.9 \%$ agree and $19.3 \%$ disagree that they could get infected at the facility, $33.2 \%$ are neutral, $11.2 \%$ strongly disagree, $5.3 \%$ strongly agree and $10.2 \%$ don't know.

\section{Response to other vaccines for rheumatologists and RD patients}

$77.2 \%$ of the rheumatology staff members do not get the influenza immunization shot regularly, $18.5 \%$ do and 3.8\% are not sure. For the RD patients, $62.3 \%$ recommend that they get the pneumococcal (pneumonia shot), $13.1 \%$ do not while $22.4 \%$ are not sure.

\section{COVID-19 infection, side effects from COVID-19 vaccine reported by rheumatology staff members}

The frequency of rheumatology staff members with confirmed or suspected infection by COVID-19 across the country was reported to be in 42.4 and $42.5 \%$ respectively. Regarding the side effects reported by the rheumatology staff members that got vaccinated are presented in Table 1. Moreover, other rare side effects have been reported including bleeding, hypercoagulability and thrombosis. Pain at injection site has also been reported. Rarely, new RD or AID reported included FMS, MS, RA, SLE and APS in one each. Most of the reported side effects were from AstraZeneca 130/187 (69.5\%) and from Sinopharm were 31/187 (16.6\%). $17(9.1 \%)$ considered both and $9(4.8 \%)$ don't know. If more vaccines were made available, the rheumatology staff members would prefer to consider the Pfizer vaccine by $40.3 \%$, 


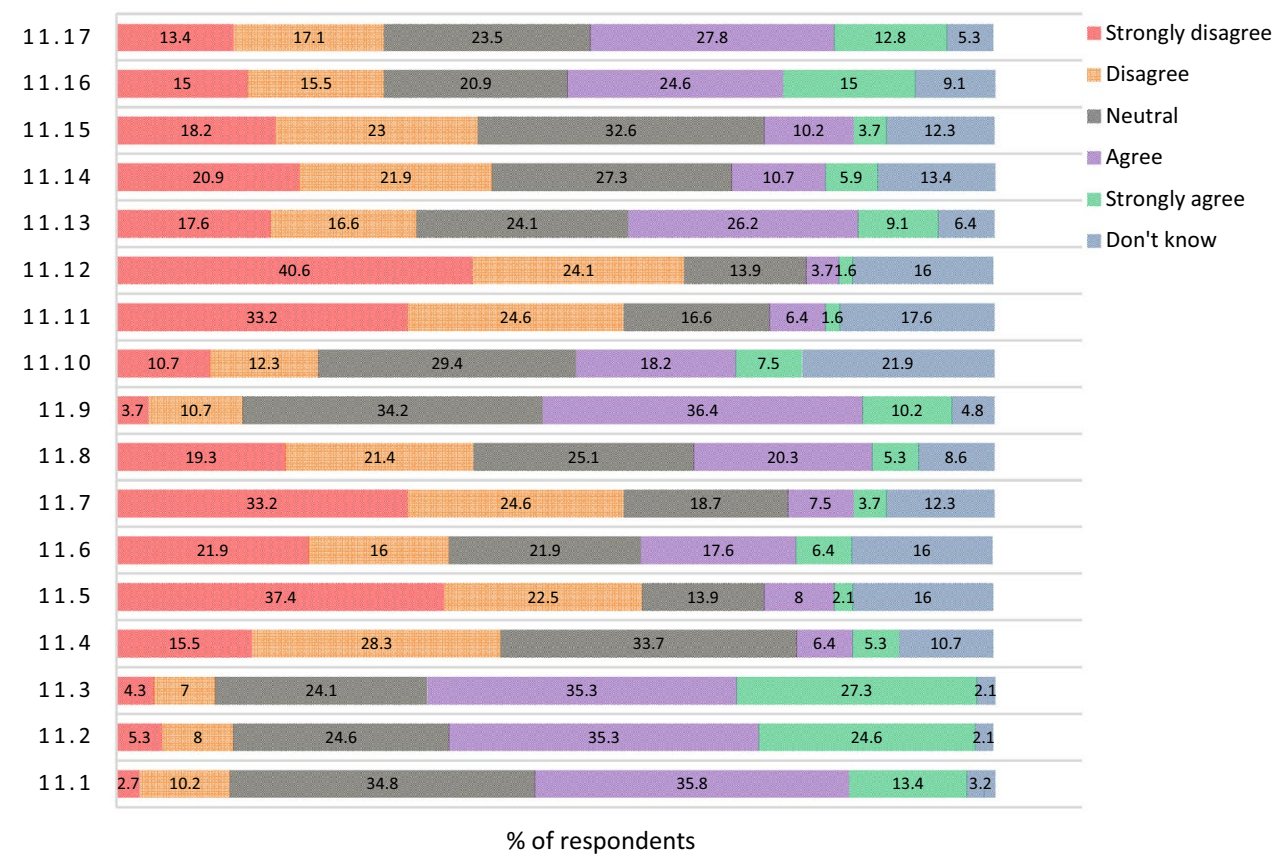

Fig. 3 The Rheumatology staff members' perspective on different COVID-19 vaccine statements: 11.1: The COVID-19 vaccines were developed too quickly; 11.2: Concern about side effects of the COVID-19 vaccine for me; 11.3: Concern about side effects of the COVID-19 vaccine for RD patients; 11.4: Unsafety of the COVID19 vaccine; 11.5: Worry about the cost of the COVID-19 vaccine; 11.6: Worry that travel will be too difficult to obtain the COVID-19 vaccine; 11.7: Worry that the appointment to receive the COVID19 vaccine is not easy for me; 11.8: Worry that the appointment to receive the COVID-19 vaccine is not easy for RD patients; 11.9: The vaccine will cause a mild course of the COVID-19 infection; 11.10: I am "provaccine"; 11.11: I never get very ill with infections so the COVID-19 vaccine is not needed for me; 11.12: The RD patients who don't get infected easily don't need the vaccine; 11.13 : Worry that the COVID-19 vaccine cause COVID-19 infection; 11.14: The COVID19 vaccine will not work as well for me; 11.15: The COVID-19 vaccine will not work as well for RD patients; 11.16: I would wait for a specific type of COVID-19 vaccine; and 11.17: I don't think the vaccine will not protect against new COVID-19 variants
Fig. 4 The Rheumatology staff members' perspective on different COVID-19 vaccine statements: 12.1: Letting infection run through the population; 12.2: COVID-19 is not dangerous for RD patients; 12.3 : The vaccine will help us return to how life was before COVID19; 12.4: Being vaccinated will alleviate the fears and worries about the pandemic; 12.5 : I want to protect myself and my family from COVID-19; 12.6: I believe in natural or traditional remedies for COVID-19 for me; 12.7: I believe in natural or traditional remedies for COVID19 for RD patients; 12.8: The vaccine will lessen the financial hardship of the pandemic; and 12.9: Wearing mask and social distancing are important ways to control COVID-19

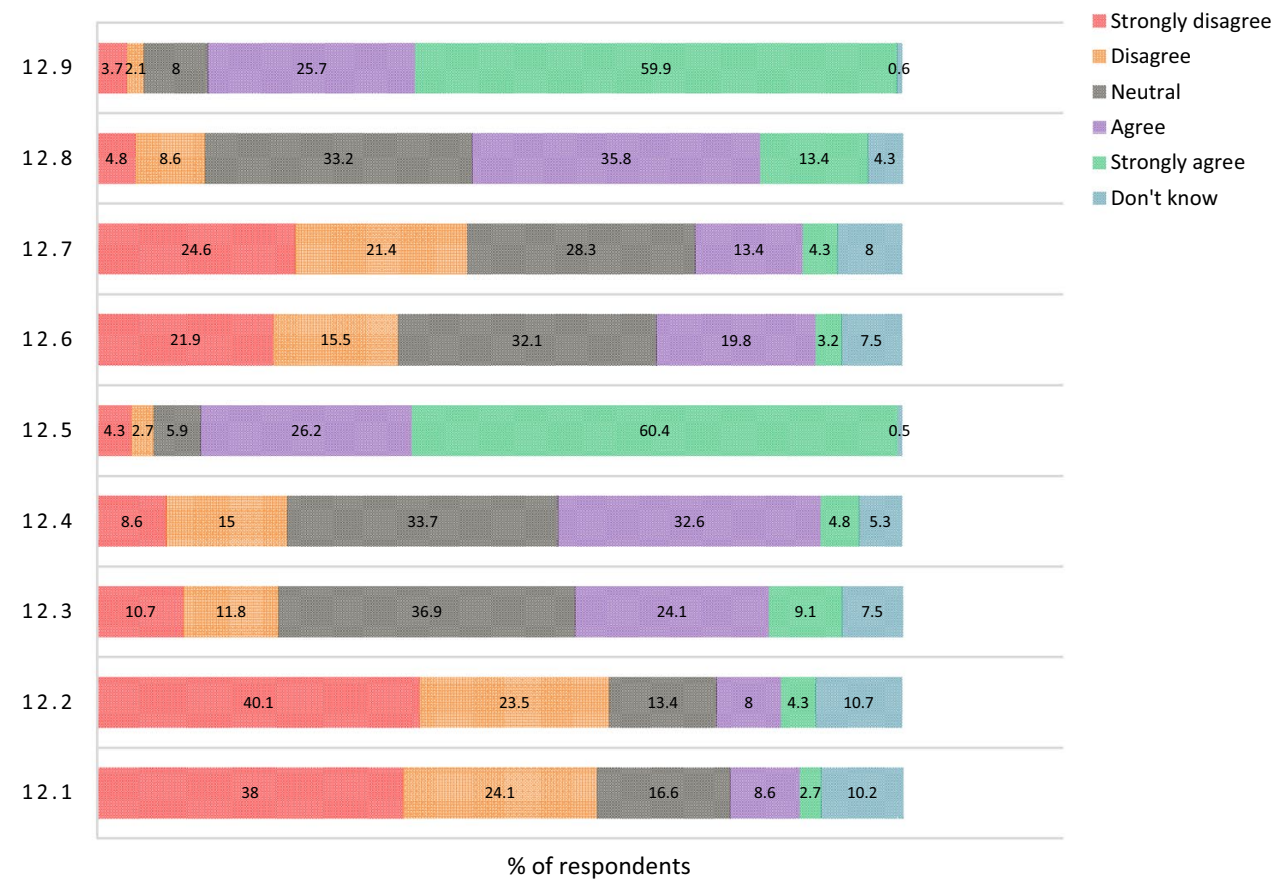


Table 1 Rating of rheumatology staff members regarding the reported side effects by those members who got vaccinated

\begin{tabular}{|c|c|c|c|c|c|c|c|c|c|c|c|c|}
\hline \multirow[t]{2}{*}{ Side effect } & \multicolumn{12}{|c|}{ Rating by rheumatology staff members $(n=187)$} \\
\hline & 0 & $<10$ & $<20$ & $<30$ & $<40$ & $<50$ & $<60$ & $<70$ & $<80$ & $<90$ & $<100$ & DK \\
\hline Anaphylaxis & 54.5 & 14.4 & 2.7 & 5.9 & 1.1 & 2.7 & 1.1 & 0.5 & 1.6 & 0 & 0 & 15.5 \\
\hline Other allergy & 36.9 & 22.5 & 10.2 & 3.7 & 3.2 & 2.7 & 1.6 & 1.6 & 0.5 & 0 & 0 & 17.1 \\
\hline Rash & 42.8 & 19.8 & 9.1 & 5.3 & 2.1 & 2.1 & 0.5 & 1.6 & 0 & 0 & 0.5 & 16 \\
\hline Fever/Chills & 4.8 & 5.9 & 8 & 7 & 7.5 & 13.9 & 6.4 & 12.3 & 13.4 & 8.6 & 5.3 & 7 \\
\hline Arthralgias & 8.5 & 7 & 7.5 & 9.1 & 9.1 & 9.6 & 8 & 8.6 & 10.2 & 7.5 & 6.4 & 8.6 \\
\hline Myalgias & 3.2 & 2.7 & 7 & 8 & 10.2 & 8.6 & 9.1 & 11.2 & 15 & 9.1 & 7.5 & 8.6 \\
\hline Fatigue & 1.6 & 3.7 & 4.3 & 6.4 & 5.9 & 11.2 & 9.6 & 8.6 & 13.9 & 20.3 & 9.1 & 5.3 \\
\hline Sleepiness & 12.8 & 9.1 & 8 & 10.2 & 5.9 & 11.2 & 7 & 5.9 & 7 & 5.9 & 3.7 & 13.4 \\
\hline Headache & 6.4 & 10.2 & 5.9 & 7 & 9.6 & 13.4 & 6.4 & 8.6 & 9.6 & 5.3 & 5.9 & 11.8 \\
\hline Nausea & 16.6 & 15 & 15 & 9.6 & 7.5 & 5.3 & 3.7 & 4.3 & 1.6 & 0.5 & 2.1 & 18.7 \\
\hline Vomiting & 28.3 & 20.9 & 9.6 & 8 & 4.3 & 3.2 & 2.1 & 1.6 & 0.5 & 0 & 0.5 & 20.9 \\
\hline Poor appetite & 16.6 & 18.7 & 11.2 & 10.2 & 5.9 & 9.6 & 2.1 & 2.1 & 0.5 & 1.1 & 2.1 & 19.8 \\
\hline Chest pain & 25.7 & 20.3 & 7.5 & 11.8 & 7 & 1.1 & 2.7 & 0.5 & 0.5 & 2.1 & 1.6 & 19.3 \\
\hline Palpitations & 24.6 & 18.2 & 10.2 & 8.6 & 4.3 & 7 & 1.1 & 1.6 & 1.6 & 0.5 & 1.6 & 20.9 \\
\hline RD flare & 26.2 & 15.5 & 5.9 & 4.8 & 5.3 & 2.1 & 1.1 & 0.5 & 1.1 & 0.5 & 0.5 & 36.4 \\
\hline New RD/AID & 42.8 & 9.6 & 3.7 & 2.7 & 2.1 & 1.1 & 2.7 & 0 & 1.6 & 0 & 0.5 & 33.2 \\
\hline
\end{tabular}

$R D$ rheumatic disease, $A I D$ autoimmune disease, $D K$ do not know
Sinopharm by $38.7 \%$, Astra by $26.5 \%$, Moderna by $11.6 \%$, Sputnik by $2.8 \%$, Johnson and Johnson by $2.8 \%$, covivax by $1.4 \%$, Sinovac by $1.1 \%$, novavax by $0.6 \%$ and $14.9 \%$ were not sure. None considered to choose the Cansino vaccine. Of the 2 available vaccines in Egypt, sinopharm is preferred for the RD patients by $55.4 \%$ and Astra by $36 \%$ while $8.6 \%$ will not provide either.

\section{COVID-19 vaccine and RD patients' disease and medications}

If a RD patient got vaccinated and consequently had a flare or worsening this will require change in treatment recommended by $53.5 \%$, while not sure by $35.8 \%$. $12.3 \%$ will not change medications. Of the greatest concerns about temporarily stopping the medications for the RD patients to give the COVID-19 vaccine were disease flare by $74.1 \%$, side effects by $13.8 \%$, withdrawal effect by $8.6 \%$, reduced efficacy when reintroduced by 6.9 and $14.9 \%$ had no concerns.

\section{Discussion}

Patients with RDs may be at higher jeopardy of infection due to many risk factors such as the disease activity, comorbidities, and immunosuppressive drugs including glucocorticoids, DMARDs and biologics in addition to the basic risk inherent to the disease itself [23]. The SARSCoV-2 infection is deemed to be a major challenge with its diverse spectrum of severity and noticeable maladaptive immune response contributing to the assorted clinical manifestations and undesirable outcomes [24]. Vaccination, an effective public-health intervention, is considered a ray of hope to eliminate the pandemic by achieving herd immunity [24]. The safety and efficacy of COVID-19 RNA vaccines in patients with autoimmune disorders is obscure, especially in those receiving immunosuppressives [3]. Sufficient information about vaccine effectiveness and safety in the immunosuppressed is warranted. Patients are willing and concerned to receive the COVID-19 vaccine, hence, rheumatologists should be prepared with the proper information before counseling their patients [20].

In the present survey, the priority to be vaccinated in RD patients goes to SLE, RA and vasculitis. SLE patients do not seem to be at higher risk of COVID-19 disease and its severity [25], however, they may be more prone to be hospitalized [7] and the disease activity may increase [26]. Patients with psoriatic arthritis (PsA) with no contraindications to vaccination should receive an mRNA-based COVID-19 vaccine onceaccessibleaccording to recent guidelines of the psoriasis foundation of America. Systemic medications for PsA are not contradictory to receiving the vaccine [27]. The German Society for Rheumatology has provided initial recommendations for patients with RDs that are purposed to answer imperative queries and to take away concerns and fears [28].

With a vaccine for SARS-CoV2 on the horizon, patients on immunosuppressives will require exceptional considerations. Immunocompromised patients were excluded from trial phases and the efficacy of the vaccine in them still needs to be scrutinized. The effects of immunosuppressives, especially methotrexate (MTX) and rituximab on a SARSCoV2 vaccine response, are undetermined [29]. However, 
it has been suggested that withholding MTX for two weeks post vaccination may enhance the response. Rituximab may impair humoral responses for $>6$ months post administration, so decisions on withholding immunosuppressives before COVID-19 vaccination will need further data [30]. Planning the vaccination of the immunocompromised patients will be needed, and considerations can be given to hold methotrexate for 2 weeks after the vaccination, and scheduling rituximab a few weeks after the vaccination until further clinical trials can answer this question [29]. Because efficacy of the vaccine is unclear both in overly immunosuppressed patients and in those with disordered immune activity, patients with RDs should continue high vigilance in social distancing, wearing masks and avoiding unnecessary contact with other individuals [31]. Still there is an unmet clinical need for the guidelines on vaccination of AIIRD patients [32].

In this study, $40.1 \%$ strongly disagree and $23.5 \%$ disagree that COVID-19 vaccine is not dangerous for RD patients. The reactogenicity of COVID-19 mRNA vaccine in patient with immune-mediated diseases has not been addressed. Immunosuppressive agents prescribed to these patients alleviateadverse effects related to vaccine immunogenicity [33]. In addition to the mechanism of molecular mimicry, mRNA vaccines may trigger a cascade of immunological events that lead to the aberrant activation of the innate and acquired immune system [33]. In a novel case series in Egypt, COVID-19 infection was likely to induce primary fibromyalgia syndrome post-COVID [34]. Still, there is uncertainty among patients with autoimmune inflammatory diseases and clinicians about the effectiveness and safety of the new vaccines. Data on the safety of COVID-19 RNA vaccines in patients with autoimmune disorders is lacking and treatments targeting the immune system could enhance or inhibit vaccine responses, depending on a patient's immune status [3]. In immunosuppressed patients, the COVID-19 mRNA vaccines lead to the production of antibodies without substantial adverse effects or disease flares [35]. With Moderna (mRNA-1273) and Pfizer/BioNTech (BNT162b2) vaccines, a reduced frequency of serious side effects $(<0.1 \%)$ has been reported.

Vaccine hesitancy seems to be highest in ethnic minority populations [19]. In this work, $35.8 \%$ agree that the vaccine was quickly made. The fact that the new vaccines were released much more quickly than the usual $12-15$ years [36] has raised considerable concern, especially among nonexperts. Previous studies reported that COVID-19 vaccine acceptance were higher among older participants [37, 38], however, in the current study, the majority of the respondents are young rheumatologists. Their concerns were about the vaccine side effects and its availability. Public acceptance for a COVID-19 vaccine seems to be waning [19]. There is growing concern that vaccine hesitancy and anti-vaccination presence will dampen the uptake of a coronavirus vaccine. Google Trends analysis has shown that throughout the pandemic the interest in a coronavirus vaccine and anti-vaccine searches has increased and peaks are found when breakthroughs are announced [39]. The relationship between vaccination uptake and perception is complex [20]. The main resistance foreseen with some of the rheumatology patients in taking the vaccine is likely to be their perception rather than scientific reasoning [20]. In Turkey, the low rate of vaccine acceptance among patients with RD, as well as general population sampling is worrying according to a web-based survey. In 320 Turkish hospital workers 52.5\% were significantly more willing to be vaccinated, $20.9 \%$ against and $26.6 \%$ unsure. Main concerns were probable side effects, unknown scientific results and having no trust. Healthcare policies should aim to implement communication, promote confidence and increase demand for COVID-19 vaccine [40].

For the RD patients, $62.3 \%$ of the rheumatology staff members recommend that they get the pneumococcal (pneumonia shot). Apart from live vaccines, all other vaccines are currently safe to use for RD patients and build-up a sufficient response to provide immunity. This creates an optimistic outlook for the new COVID-19 vaccine and its anticipated therapeutic effect [20]. Some evidence indicates that immunosuppressive therapy inhibits humoral response to the influenza, and pneumococcal vaccines. The degree to which this will translate to impaired COVID-19 vaccine responses is unclear [30]. It is favorable that subjects with a dysfunctional immune response receive the COVID-19 mRNA vaccine only if the benefits outweigh the risks and after a careful personalized assessment [33].

About one third of patients are neutral to whether the vaccine will lead to a disease flare, $21.9 \%$ disagree, $12.8 \%$ strongly disagree. As it will take months before all RD patients have access to the vaccines, current actions taken to enhance the safety and efficacy of the vaccines may reactivate the underlying RD [28]. Many factors at play provide challenges to the response of patients to SARS-CoV-2 vaccines and in the setting of immune dysfunction, adaption in vaccination strategies is requiredto achieve adequate and durable immune responses. In the future as a consequence to vaccination more information is required on how to manage these drugs and monitor the patient's immunity [41].

The American College of Rheumatology COVID-19 Vaccine Clinical Guidance Task Force has just released a guidance document attempting to shed light on the complexities of vaccinating patients with autoimmune RDs. The main guidance statements are patients with RDs should receive the COVID-19 vaccines and should be prioritized before the general population. Specific submissions regarding the timing and changes to commonly used medications are included with the caveat that these need to be individualized [42]. 
The ACR guidelines provide direction on how to best use COVID-19 vaccines and to facilitate implementation of vaccination strategies for RMD patients [43]. Among the suggested recommendations, COVID-19 vaccinations should preferably be made during remission. Low-degree immunosuppression may not interfere with antibody response to vaccines. Ideally, vaccinations should be made before the initiation of any biological DMARDs. Timing of vaccination is especially important in the case of rituximab [44]. A task force of the Korean College of Rheumatology (KCR) formulated a set of vaccination guidance. Every patient with autoimmune/autoinflammatory RDs (AIIRD) should receive one of the available COVID-19 vaccines unless contraindicated. Patients should continue immunosuppressive treatment for their underlying AIIRD. Corticosteroids should be reduced to the lowest dose possible without aggravating the AIIRD. To improve the vaccine response, MTX can be withheld for 1-2 weeks after each vaccination [45].

Among the study limitations is that not all staff members participated.The present perspectives represent rheumatologists from academic centers, but lacking participation of rheumatologists serving in other sectors as the ministry of health and private practices may further limit the vision concerning RD patients. Furthermore, this survey is a reflection of the perspective at a certain time that may change according to newly emerging situations.

\section{Conclusion}

We identified a low rate of acceptance ofCOVID-19 vaccines among rheumatology staff members. We believe these results and specifically the low rate of acceptability is alarming to Egyptian health authorities. Further studies on the causes of low acceptance rate and the need to increase the trusted source regarding information about COVID-19 vaccines are required. Systematic interventions are required by public-health authorities to reduce the levels of vaccines' hesitancy and improve their acceptance. As rheumatic disease patients in Egypt were not systematically provided the vaccine till present and most rheumatologists are willing to, making the vaccine available by the government for high priority groups could as well enhance vaccines acceptance. Further studies to investigate any possible side effects, on a large scale of RD patients in Egypt, are warranted in the near future.

Supplementary Information The online version contains supplementary material available at https://doi.org/10.1007/s00296-021-04941-0.

Acknowledgements Egyptian College of Rheumatology Collaborators: Hala A Raafat (Cairo), Samar M Fawzy (Cairo), Iman ElGazzar (Cairo), Nahla N Eesa (Cairo),Soha E Ibrahim (Ain Shams), Reem El-Mallah (Ain Shams), NermeenNoshy (Ain Shams), Esam Abualfadl
(Sohag), Osama S Abousehly (Sohag), Dina F El-Essawi (AEA), Eman Hassan (Alexandria), Ehab Saad (Qena), Emtethal A Said (Benha), Samah I Nasef (Suez Canal).

The authors would like to acknowledge the 187 Rheumatology Colleagues across the universities of Egypt for their active participation and filling out the form. A special word of thanks to Julia F Simard, professor of epidemiology and population health, Stanford School of Medicine and a principal investigator in the GRA, for her encouragement to actively participate in the international gathering and follow those step; and for Jonathan S Hausmann, professor of Medicine (Rheumatology), Harvard Medical School, GRA patient registries collaboration lead for his request to collaborate in the GRA patients vaccine survey translation in to Arabic language.

Funding This research did not receive any specific grant from funding agencies in the public, commercial, or not-for-profit sectors.

\section{Declarations}

Conflict of interest None.

\section{References}

1. Misra DP, Agarwal V, Gasparyan AY, Zimba O (2020) Rheumatologists' perspective on coronavirus disease 19 (COVID-19) and potential therapeutic targets. Clin Rheumatol. https://doi.org/10. 1007/s10067-020-05073-9

2. Ceribelli A, Motta F, De Santis M et al (2020) Recommendations for coronavirus infection in rheumatic diseases treated with biologic therapy. J Autoimmun 109:102442. https://doi.org/10.1016/j. jaut.2020.102442

3. Tang W, Askanase AD, Khalili L, Merrill JT (2021) SARS-CoV-2 vaccines in patients with SLE. Lupus Sci Med 8:e000479. https:// doi.org/10.1136/lupus-2021-000479

4. Egypt: WHO Coronavirus Disease (COVID-19) Dashboard With Vaccination Data. https://covid19.who.int. Accessed 13 Jun 2021

5. COVID Live Update: 176,484,692 Cases and 3,812,240 Deaths from the Coronavirus - Worldometer. https://www.worldometers. info/coronavirus/. Accessed 13 Jun 2021

6. Guan W, Ni Z, Hu Y et al (2020) Clinical characteristics of coronavirus disease 2019 in China. N Engl J Med 382:1708-1720. https://doi.org/10.1056/NEJMoa2002032

7. de Almeida-Pititto B, Dualib PM, Zajdenverg L et al (2020) Severity and mortality of COVID 19 in patients with diabetes, hypertension and cardiovascular disease: a meta-analysis. Diabetol Metab Syndr 12:1-12. https://doi.org/10.1186/s13098-020-00586-4

8. Kabeerdoss J, Danda D (2020) Understanding immunopathological fallout of human coronavirus infections including COVID-19: Will they cross the path of rheumatologists? Int J Rheum Dis 23:998-1008. https://doi.org/10.1111/1756-185X.13909

9. Gheita TA, Salem MN, Eesa NN et al (2020) Rheumatologists' practice during the Coronavirus disease 2019 (COVID-19) pandemic: a survey in Egypt. Rheumatol Int 40:1599-1611. https:// doi.org/10.1007/s00296-020-04655-9

10. Abualfadl E, Ismail F, Shereef RRE et al (2021) Impact of COVID-19 pandemic on rheumatoid arthritis from a Multi-Centre patient-reported questionnaire survey: influence of gender, ruralurban gap and north-south gradient. Rheumatol Int 41:345-353. https://doi.org/10.1007/s00296-020-04736-9

11. Batu ED, Özen S (2020) Implications of COVID-19 in pediatric rheumatology. Rheumatol Int 40:1193-1213. https://doi.org/10. 1007/s00296-020-04612-6 
12. Gianfrancesco M, Hyrich KL, Al-Adely S et al (2020) Characteristics associated with hospitalisation for COVID-19 in people with rheumatic disease: data from the COVID-19 Global Rheumatology Alliance physician-reported registry. Ann Rheum Dis 79:859-866. https://doi.org/10.1136/annrheumdis-2020-217871

13. Akiyama S, Hamdeh S, Micic D, Sakuraba A (2020) Prevalence and clinical outcomes of COVID-19 in patients with autoimmune diseases: a systematic review and meta-analysis. Ann Rheum Dis 80:384-391. https://doi.org/10.1136/annrheumdis-2020-218946

14. Izda V, Jeffries MA, Sawalha AH (2021) COVID-19: a review of therapeutic strategies and vaccine candidates. Clin Immunol 222:108634. https://doi.org/10.1016/j.clim.2020.108634

15. Benucci M, Infantino M, Marotto D et al (2021) Vaccination against SARS-CoV-2 in patients with rheumatic diseases: doubts and perspectives. Clin Exp Rheumatol 39:196-202

16. Strizova Z, Smetanova J, Bartunkova J, Milota T (2021) Principles and challenges in anti-COVID-19 vaccine development. Int Arch Allergy Immunol 182:339-349. https://doi.org/10.1159/00051 4225

17. Speiser DE, Bachmann MF (2020) COVID-19: mechanisms of vaccination and immunity. Vaccines 8:404. https://doi.org/10. 3390/vaccines8030404

18. Atzeni F, Masala IF, Rodríguez-Carrio J et al (2021) The rheumatology drugs for COVID-19 management: which and when? J Clin Med 10:783. https://doi.org/10.3390/jcm10040783

19. Glover RE, Urquhart R, Lukawska J, Blumenthal KG (2021) Vaccinating against covid-19 in people who report allergies. BMJ 372:n120. https://doi.org/10.1136/bmj.n120

20. Byravan S, Fardanesh A, Tahir H, Moorthy A (2021) Emerging COVID-19 vaccines: a rheumatology perspective. Int J Rheum Dis 24:144-146. https://doi.org/10.1111/1756-185X.14048

21. Gaur PS, Zimba O, Agarwal V, Gupta L (2020) Reporting survey based studies—a primer for authors. J Korean Med Sci 35:e398. https://doi.org/10.3346/jkms.2020.35.e398

22. Patient Experience Survey Updates I The COVID-19 Global Rheumatology Alliance. https://rheum-covid.org/patient-exper ience-survey-update

23. Sarzi-Puttini P, Marotto D, Antivalle M et al (2020) How to handle patients with autoimmune rheumatic and inflammatory bowel diseases in the COVID-19 era: an expert opinion. Autoimmun Rev 19:102574. https://doi.org/10.1016/j.autrev.2020.102574

24. Velikova T, Georgiev T (2021) SARS-CoV-2 vaccines and autoimmune diseases amidst the COVID-19 crisis. Rheumatol Int. https://doi.org/10.1007/s00296-021-04792-9

25. Favalli EG, Gerosa M, Murgo A, Caporali R (2021) Are patients with systemic lupus erythematosus at increased risk for COVID19? Ann Rheum Dis 80:e25-e25. https://doi.org/10.1136/annrh eumdis-2020-217787

26. Raghavan S, Gonakoti S, Asemota IR, Mba B (2020) A case of systemic Lupus Erythematosus flare triggered by severe Coronavirus disease 2019. J Clin Rheumatol 26:234-235. https://doi.org/ 10.1097/RHU.0000000000001531

27. Gelfand JM, Armstrong AW, Bell S et al (2021) National Psoriasis Foundation COVID-19 Task Force guidance for management of psoriatic disease during the pandemic: version 2-advances in psoriatic disease management, COVID-19 vaccines, and COVID-19 treatments. J Am Acad Dermatol 84:1254-1268. https://doi.org/ 10.1016/j.jaad.2020.12.058

28. Schulze-Koops H, Specker C, Skapenko A (2021) Vaccination of patients with inflammatory rheumatic diseases against SARSCoV-2: considerations before widespread availability of the vaccines. RMD Open 7:e001553. https://doi.org/10.1136/rmdop en-2020-001553

29. Sonani B, Aslam F, Goyal A et al (2021) COVID-19 vaccination in immunocompromised patients. Clin Rheumatol. https://doi.org/ 10.1007/s10067-020-05547-w
30. Arnold J, Winthrop K, Emery P (2021) COVID-19 vaccination and antirheumatic therapy. Rheumatology. https://doi.org/10. 1093/rheumatology/keab223

31. CDC (2020) Coronavirus Disease 2019 (COVID-19). In: Cent. Dis. Control Prev. https://www.cdc.gov/coronavirus/2019-ncov/ index.html

32. Furer V, Rondaan C, Agmon-Levin N et al (2021) Point of view on the vaccination against COVID-19 in patients with autoimmune inflammatory rheumatic diseases. RMD Open 7:e001594. https:// doi.org/10.1136/rmdopen-2021-001594

33. Talotta R (2021) Do COVID-19 RNA-based vaccines put at risk of immune-mediated diseases? In reply to "potential antigenic cross-reactivity between SARS-CoV-2 and human tissue with a possible link to an increase in autoimmune diseases." Clin Immunol Orlando Fla 224:108665. https://doi.org/10.1016/j.clim.2021. 108665

34. Gheita TA, Fathi HM, ElAdle SS, Eesa NN, Hammam NH (2021) Coronavirus disease 2019 (COVID-19) an emerging trigger for primary fibromyalgia syndrome: a tale of three cases postCOVID-19. Int J Clin Rheumatol 16:129

35. Geisen UM, Berner DK, Tran F et al (2021) Immunogenicity and safety of anti-SARS-CoV-2 mRNA vaccines in patients with chronic inflammatory conditions and immunosuppressive therapy in a monocentric cohort. Ann Rheum Dis. https://doi.org/10.1136/ annrheumdis-2021-220272

36. Han S (2015) Clinical vaccine development. Clin Exp Vaccine Res 4:46-53. https://doi.org/10.7774/cevr.2015.4.1.46

37. Lazarus JV, Ratzan SC, Palayew A et al (2021) A global survey of potential acceptance of a COVID-19 vaccine. Nat Med 27:225-228. https://doi.org/10.1038/s41591-020-1124-9

38 Malik AA, McFadden SM, Elharake J, Omer SB (2020) Determinants of COVID-19 vaccine acceptance in the US. EClinicalMedicine 26:100495. https://doi.org/10.1016/j.eclinm.2020.100495

39. Pullan S, Dey M (2021) Vaccine hesitancy and anti-vaccination in the time of COVID-19: a Google Trends analysis. Vaccine 39:1877-1881. https://doi.org/10.1016/j.vaccine.2021.03.019

40. Yurttas B, Poyraz BC, Sut N et al (2021) Willingness to get the COVID-19 vaccine among patients with rheumatic diseases, healthcare workers and general population in Turkey: a webbased survey. Rheumatol Int 41:1105-1114. https://doi.org/10. 1007/s00296-021-04841-3

41. Ferro F, Elefante E, Puxeddu I et al (2020) COVID-19: the new challenge for rheumatologists. Clin Exp Rheumatol 38:373-382

42. ACR COVID-19 Vaccine Clinical Guidance Task Force. COVID19 vaccine clinical guidance summary for patients with rheumatic and musculoskeletal diseases. https://www.rheumatology.org/ Portals/0/Files/COVID-19-Vaccine-Clinical-Guidance_Rheum atic-Diseases-Summary.pdf

43. Curtis JR, Johnson SR, Anthony DD et al (2021) American College of Rheumatology guidance for COVID-19 vaccination in patients with rheumatic and musculoskeletal diseases: version 1. Arthritis Rheumatol. https://doi.org/10.1002/art.41734

44. Soy M, Keser G, Atagunduz P et al (2021) A practical approach for vaccinations including COVID-19 in autoimmune/autoinflammatory rheumatic diseases: a non-systematic review. Clin Rheumatol 22:1-13. https://doi.org/10.1007/s10067-021-05700-z

45. Park JK, Lee EB, Shin K et al (2021) COVID-19 vaccination in patients with autoimmune inflammatory rheumatic diseases: clinical guidance of the Korean College of Rheumatology. J Korean Med Sci 36:e95. https://doi.org/10.3346/jkms.2021.36.e95

Publisher's Note Springer Nature remains neutral with regard to jurisdictional claims in published maps and institutional affiliations. 


\section{Authors and Affiliations}

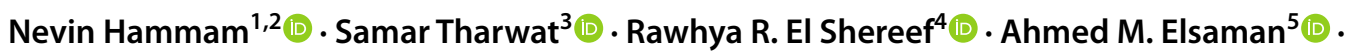

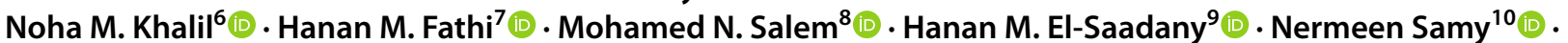

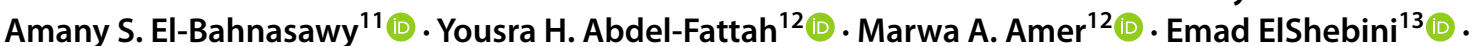

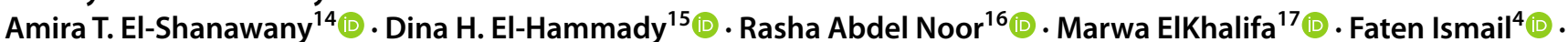

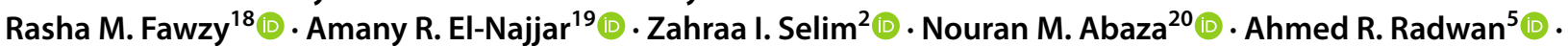

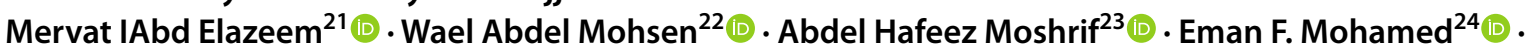

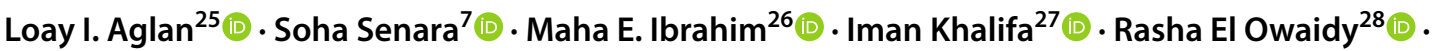

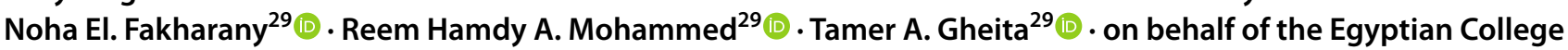
of Rheumatology (ECR) COVID-19 Study Group

1 Rheumatology Department, Faculty of Medicine, Assiut University, Assiut, Egypt

2 Rheumatology Department, University of California San Francisco (UCSF), San Francisco, CA, USA

3 Rheumatology Unit, Internal Medicine, Mansoura University, Dakahlia, Egypt

4 Rheumatology Department, Faculty of Medicine, Minia University, Minia, Egypt

5 Rheumatology Department, Faculty of Medicine, Sohag University, Sohag, Egypt

6 Internal Medicine Department, Rheumatology Unit, Faculty of Medicine, Cairo University, Cairo, Egypt

7 Rheumatology Department, Faculty of Medicine, Fayoum University, Fayoum, Egypt

8 Internal Medicine Department, Rheumatology Unit, Faculty of Medicine, Beni-Suef University, Beni-Suef, Egypt

9 Rheumatology Department, Faculty of Medicine, Tanta University, Gharbia, Egypt

10 Internal Medicine Department, Rheumatology Unit, Faculty of Medicine, Ain Shams University, Cairo, Egypt

11 Rheumatology Department, Faculty of Medicine, Mansoura University, Dakahlia, Egypt

12 Rheumatology Department, Faculty of Medicine, Alexandria University, Alexandria, Egypt

13 Internal Medicine Department, Rheumatology Unit, Menoufia University, Menoufia, Egypt

14 Rheumatology Department, Faculty of Medicine, Menoufia University, Menoufia, Egypt

15 Rheumatology Department, Faculty of Medicine, Helwan University, Cairo, Egypt
16 Internal Medicine Department, Rheumatology Unit, Tanta University, Gharbia, Egypt

17 Internal Medicine Department, Rheumatology Unit, Faculty of Medicine, Alexandria University, Alexandria, Egypt

18 Rheumatology Department, Faculty of Medicine, Benha University, Kalubia, Egypt

19 Rheumatology Department, Faculty of Medicine, Zagazig University, Sharkia, Egypt

20 Rheumatology Department, Faculty of Medicine, Ain Shams University, Cairo, Egypt

21 Rheumatology Department, Faculty of Medicine, Beni-Suef University, Beni-Suef, Egypt

22 Rheumatology Department, Faculty of Medicine, South Valley University, Qena, Egypt

23 Rheumatology Department, Faculty of Medicine, Al-Azhar University, Assiut, Egypt

24 Internal Medicine Department, Rheumatology Unit, Faculty of Medicine (Girls), Al-Azhar University, Cairo, Egypt

25 Rheumatology Department, Faculty of Medicine, Aswan University, Aswan, Egypt

26 Rheumatology Department, Faculty of Medicine, Suez-Canal University, Ismailia, Egypt

27 Pediatrics Department, Rheumatology and Nephrology Unit, Helwan University, Cairo, Egypt

28 Pediatrics Department, Rheumatology Unit, Ain Shams University, Cairo, Egypt

29 Rheumatology Department, Faculty of Medicine, Cairo University, Cairo, Egypt 